\title{
Clinical usefulness of gefitinib for non-small-cell lung cancer with a double epidermal growth factor receptor mutation
}

\author{
TAKEFUMI OIKAWA ${ }^{1}$, TATSUO OHIRA ${ }^{2}$, KEISHI OTANI ${ }^{2}$, \\ MASARU HAGIWARA $^{2}$, CHIMORI KONAKA ${ }^{1}$ and NORIHIKO IKEDA ${ }^{2}$ \\ ${ }^{1}$ Chemotherapy Research Institute, Kaken Hospital, Ichikawa, Chiba 272-0827; \\ ${ }^{2}$ Department of Surgery, Tokyo Medical University, Tokyo 160-0023, Japan
}

Received September 15, 2014; Accepted October 23, 2014

DOI: $10.3892 / \mathrm{mco} .2014 .455$

\begin{abstract}
The aim of this study was to investigate whether the pattern of epidermal growth factor receptor (EGFR) gene mutations affects sensitivity to gefitinib treatment. We investigated 44 surgically resected non-small-cell lung cancer (NSCLC) specimens obtained between 2001 and 2012 at the Tokyo Medical University Hospital. The specimens were obtained from patients treated with gefitinib as 1st-, 2nd-, or 3rd-line therapy for postoperative recurrent NSCLC. We detected EGFR mutations using the cycleave PCR technique. In addition, the specimens from non-responders were stained with antibodies against hepatocyte growth factor receptor (HGFR; MET) and hepatocyte growth factor (HGF). We assessed the progression of non-responders over a period of 2 months. Intermediate responders were considered to be patients who responded (exhibiting at least stable disease) to gefitinib therapy for 3-11 months, while long-term responders were defined as those who responded to gefitinib therapy for $>12$ months. The NSCLCs were histologically classified as 43 adenocarcinomas and one large-cell neuroendocrine carcinoma. One patient had an exon 18 point mutation, 23 an exon 19 deletion, 2 an exon 20 point mutation, 16 an exon 21 point mutation and 2 patients had both exon 20 and 21 point mutations. There were 4 non-responders, including the 2 patients with exon 20 mutation, 25 intermediate responders (including 10 patients under ongoing treatment) and 15 long-term responders ( 2 of whom are under ongoing treatment), including the 2 patients with both exon 20 and 21 mutations. Of the specimens obtained from non-responders, 3 stained with the anti-MET antibody and 1 stained with the anti-HGF antibody. Therefore, NSCLC with exon 20 mutation may respond to gefitinib treatment in the presence of an additional EGFR mutation.
\end{abstract}

Correspondence to: Professor Norihiko Ikeda, Department of Surgery, Tokyo Medical University, 6-7-1 Nishi-shinjuku, Shinjuku-ku, Tokyo 160-0023, Japan

E-mail:ikeda@wd5.so-net.ne.jp

Key words: double mutation, T790M, gefitinib, non-small-cell lung cancer

\section{Introduction}

Somatic mutations in the tyrosine kinase (TK) domain of epidermal growth factor receptor (EGFR) gene have been reported in patients with non-small-cell lung cancer (NSCLC). Certain mutations in the EGFR gene, such as leucine-to-arginine substitution at amino acid position 858 (L858R) in exon 21 or deletions in exon 19, are highly correlated with sensitivity to EGFR-TK inhibitors (TKIs) $(1,2)$. The EGFR-TKIs gefitinib and erlotinib are effective in the treatment of EGFR-mutant NSCLC; however, there are also cases of EGFR-mutant NSCLCs exhibiting resistance to EGFR-TKI treatment. EGFR-TKIs have been shown to achieve a response in $\sim 80 \%$ NSCLC patients with EGFR mutations, indicating that $\sim 20 \%$ of NSCLC patients with EGFR mutation are unresponsive to this treatment (3).

A threonine-to-methionine substitution at amino acid position 790 (T790M) in exon 20 was reportedly associated with acquired resistance to EGFR-TKIs $(4,5)$. In addition, hepatocyte growth factor receptor (HGFR; MET) gene amplification was reportedly associated with acquired resistance to EGFR-TKIs (6), while hepatocyte growth factor (HGF)-mediated MET activation was reported as the mechanism underlying EGFR-TKI resistance in lung cancer with EGFR-activating mutations (7). However, these studies were not pertaining to resistance, but rather investigating acquired resistance to EGFR-TKIs.

It was recently reported that pretreatment of NSCLC with T790M shortens the duration of response to EGFR-TKIs (9-12). However, over the last few years, we have observed long progression-free survival (PFS) in patients with T790M.

In this study, we aimed to investigate the pattern of EGFR mutations in NSCLC that affects sensitivity to EGFR-TKIs, determine the cause of shortened EGFR-TKI response duration and determine the correlation between resistance to EGFR-TKIs and phosphorylated MET or HGF expression.

\section{Materials and methods}

Patients and specimens. We investigated 44 surgically resected NSCLCs between 2001 and 2012. The specimens were obtained from patients treated with gefitinib as 1st-, 2nd-, or 3rd-line therapy for postoperative recurrent NSCLC. 
A

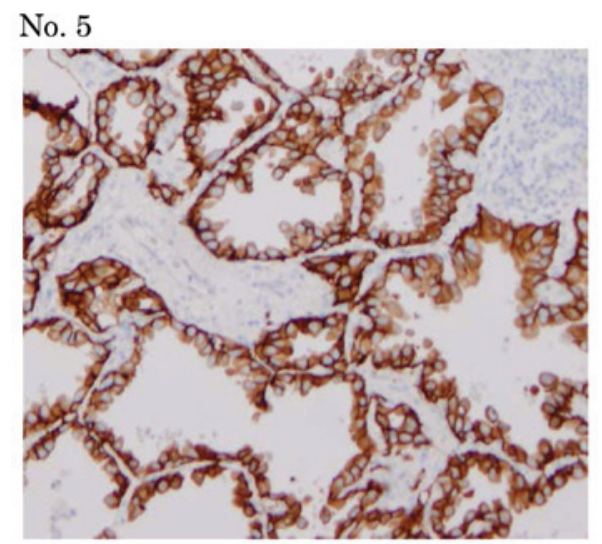

No. 26

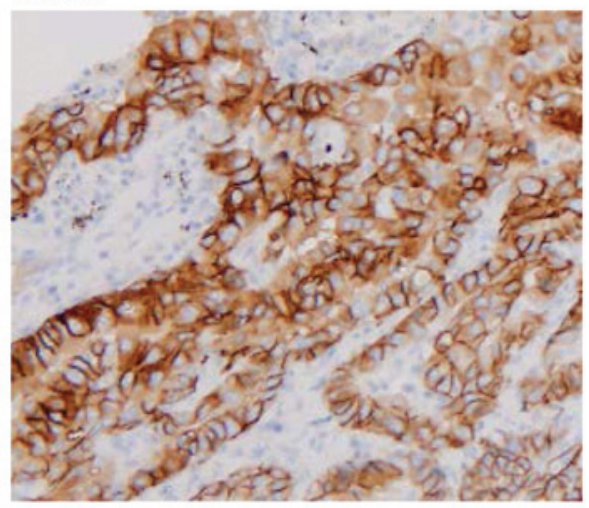

\section{B}

No. 5

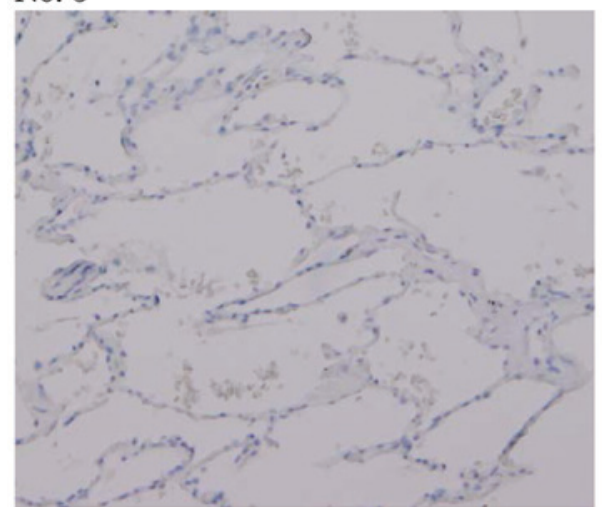

No. 26

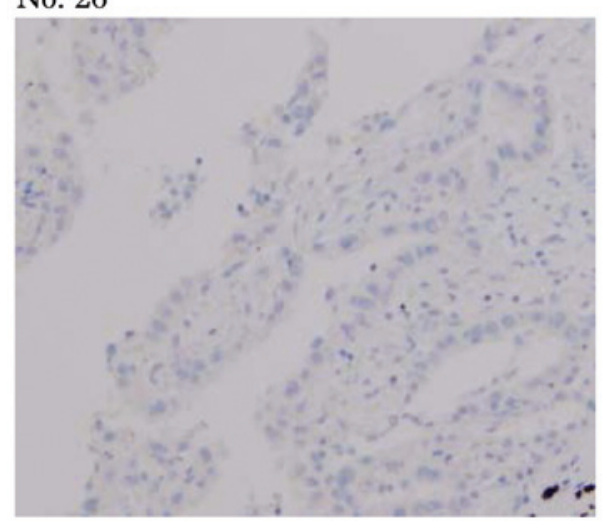

No. 25

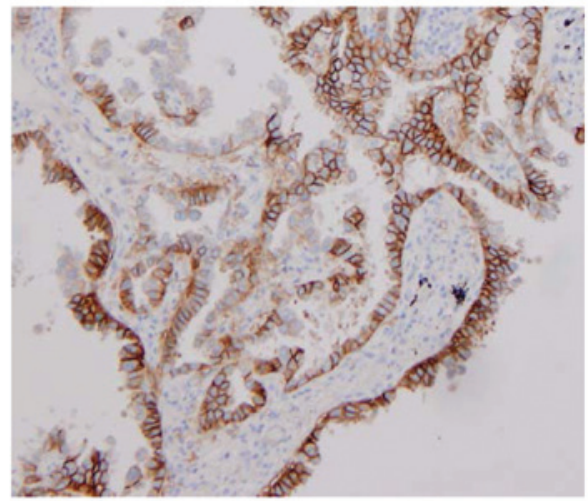

No. 32

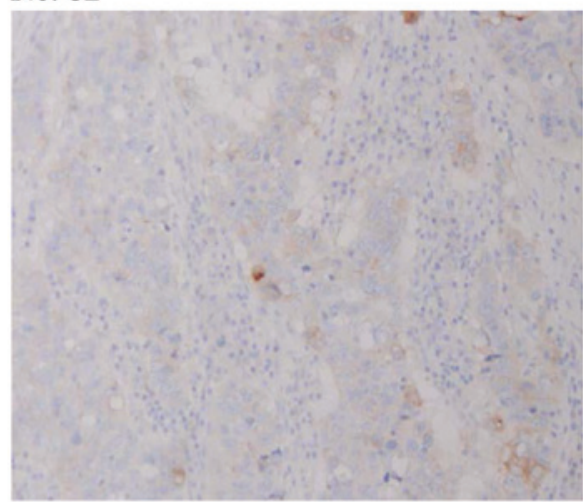

No. 25

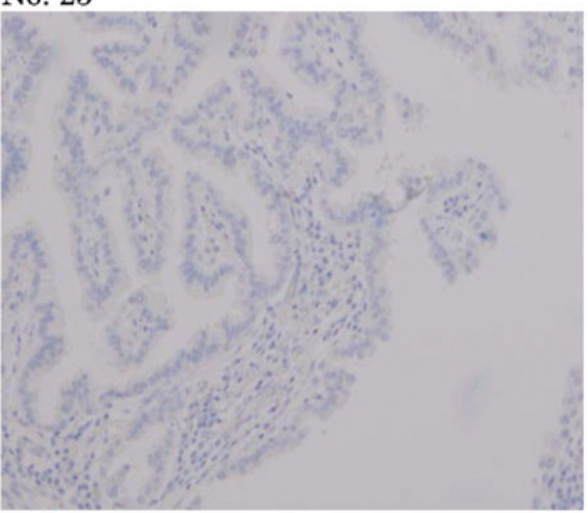

No. 32

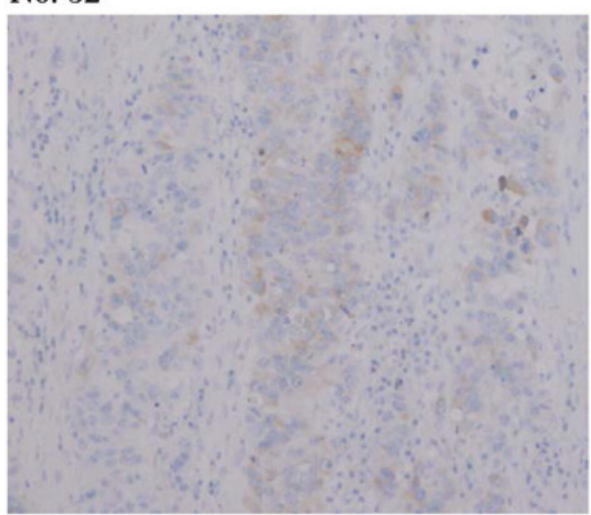

Figure 1. Immunohistochemical staining of non-small-cell lung cancer specimens. (A) The immunohistochemical staining for hepatocyte growth factor receptor (HGFR; MET) exhibited strong reactivity in the cell membranes of specimens no. 5,25 and 26, whereas there was no reactivity with MET in specimen no. 32. (B) The immunohistochemical staining for HGF exhibited strong reactivity in the cell membranes of specimen no. 32, whereas there was no reactivity with HGF in specimens no. 5, 25, and 26. 
Table I. Patient characteristics and response to gefitinib.

\begin{tabular}{|c|c|c|c|c|c|c|}
\hline Patients & Gender & $\begin{array}{c}\text { Age } \\
\text { (years) }\end{array}$ & $\begin{array}{l}\text { Histological } \\
\text { type }\end{array}$ & Exon & Lobectomy & Response $^{a}$ \\
\hline 1 & $\mathrm{M}$ & 46 & AdenoCa & 18 & Total & 2 \\
\hline 2 & $\mathrm{~F}$ & 73 & AdenoCa & 19 & Total & 2 \\
\hline 3 & $\mathrm{~F}$ & 59 & AdenoCa & 19 & Total & 3 \\
\hline 4 & $\mathrm{~F}$ & 71 & AdenoCa & 19 & Total & 3 \\
\hline 5 & $\mathrm{~F}$ & 54 & AdenoCa & 19 & Total & 1 \\
\hline 6 & $\mathrm{M}$ & 63 & AdenoCa & 19 & Total & 2 \\
\hline 7 & $\mathrm{~F}$ & 59 & AdenoCa & 19 & Total & 2 \\
\hline 8 & $\mathrm{M}$ & 78 & AdenoCa & 19 & Partial & 2 \\
\hline 9 & $\mathrm{~F}$ & 68 & AdenoCa & 19 & Partial & 2 \\
\hline 10 & $\mathrm{~F}$ & 71 & AdenoCa & 19 & Total & 2 \\
\hline 11 & $\mathrm{~F}$ & 73 & AdenoCa & 19 & Total & 2 \\
\hline 12 & M & 66 & AdenoCa & 19 & Total & 2 \\
\hline 13 & $\mathrm{~F}$ & 51 & AdenoCa & 19 & Total & 2 \\
\hline 14 & M & 61 & AdenoCa & 19 & Total & 2 \\
\hline 15 & $\mathrm{M}$ & 47 & AdenoCa & 19 & Total & 2 \\
\hline 16 & $\mathrm{M}$ & 60 & AdenoCa & 19 & Total & 3 \\
\hline 17 & $\mathrm{~F}$ & 54 & AdenoCa & 19 & Total & 3 \\
\hline 18 & $\mathrm{M}$ & 79 & AdenoCa & 19 & Total & 2 \\
\hline 19 & $\mathrm{~F}$ & 27 & AdenoCa & 19 & Total & 2 \\
\hline 20 & $\mathrm{M}$ & 75 & AdenoCa & 19 & Total & 2 \\
\hline 21 & $\mathrm{~F}$ & 61 & AdenoCa & 19 & Total & 3 \\
\hline 22 & $\mathrm{~F}$ & 77 & AdenoCa & 19 & Total & 3 \\
\hline 23 & $\mathrm{~F}$ & 56 & AdenoCa & 19 & Total & 3 \\
\hline 24 & $\mathrm{~F}$ & 55 & AdenoCa & 19 & Total & 3 \\
\hline 25 & $\mathrm{~F}$ & 55 & AdenoCa & 20 & Total & 1 \\
\hline 26 & M & 72 & AdenoCa & 20 & Total & 1 \\
\hline 27 & $\mathrm{~F}$ & 71 & AdenoCa & 21 & Total & 2 \\
\hline 28 & $\mathrm{~F}$ & 66 & AdenoCa & 21 & Total & 3 \\
\hline 29 & $\mathrm{M}$ & 69 & AdenoCa & 20,21 & Total & 3 \\
\hline 30 & $\mathrm{M}$ & 64 & AdenoCa & 20,21 & Total & 3 \\
\hline 31 & $\mathrm{M}$ & 72 & AdenoCa & 21 & Total & 3 \\
\hline 32 & $\mathrm{~F}$ & 60 & AdenoCa & 21 & Total & 1 \\
\hline 33 & $\mathrm{~F}$ & 78 & Large-cell Ca & 21 & Total & 2 \\
\hline 34 & M & 57 & AdenoCa & 21 & Total & 2 \\
\hline 35 & $\mathrm{M}$ & 78 & AdenoCa & 21 & Total & 2 \\
\hline 36 & $\mathrm{~F}$ & 76 & AdenoCa & 21 & Total & 2 \\
\hline 37 & $\mathrm{M}$ & 65 & AdenoCa & 21 & Total & 3 \\
\hline 38 & $\mathrm{~F}$ & 60 & AdenoCa & 21 & Total & 2 \\
\hline 39 & $\mathrm{M}$ & 39 & AdenoCa & 21 & Total & 2 \\
\hline 40 & $\mathrm{~F}$ & 57 & AdenoCa & 21 & Total & 2 \\
\hline 41 & $\mathrm{M}$ & 70 & AdenoCa & 21 & Total & 2 \\
\hline 42 & $\mathrm{M}$ & 42 & AdenoCa & 21 & Total & 3 \\
\hline 43 & $\mathrm{~F}$ & 62 & AdenoCa & 21 & Total & 3 \\
\hline 44 & $\mathrm{~F}$ & 73 & AdenoCa & 21 & Total & 2 \\
\hline
\end{tabular}

${ }^{\mathrm{a}}$, No response; 2, intermediate response; 3, long-term response. M, male; F, female; Ca, carcinoma.

The NSCLCs were histologically classified as 43 adenocarcinomas and 1 large-cell neuroendocrine carcinoma. The patients included 19 men and 25 women, aged 27-78 years (mean age, 63.0 years).
Immunostaining. We detected EGFR mutations in matching formalin-fixed, paraffin-embedded tissue samples using the cycleave PCR technique (SRL Inc., Tokyo, Japan). We used an anti-MET rabbit monoclonal antibody (clone SP44; 
Table II. Type of EGFR mutation and response to gefitinib.

Type of mutation

\begin{tabular}{lcccc}
\cline { 2 - 4 } Type of response & Exon 18 & Exon 19 & Exon 20 & Exon 2 \\
\hline Non-responders & 0 & 1 & 2 & 1 \\
Intermediate responders & 1 & 14 & 0 & 10 \\
Long-term responders & 0 & 8 & $2^{\text {a }}$ & $7^{\text {a }}$
\end{tabular}

ancluding 2 patients with both exon 20 and 21 mutations. EGFR, epidermal growth factro receptor.

cat no. 518-108830; Ventana Medical Systems, Inc., Tucson, AZ, USA) for MET staining and a goat polyclonal anti-human HGF antibody (cat no. 36073; LifeSpan BioSciences, Inc., Seattle, WA, USA) at a 1:40 dilution for HGF staining. Immunostaining for MET and HGF was performed using the Ventana System (Ventana Medical Systems, Inc, Harvard, MA, USA).

Type of response to gefitinib. We assessed the progression of non-responders to gefitinib treatment over a 2-month period. Intermediate responders included patients who responded (exhibiting at least stable disease) to gefitinib for 3-11 months. Long-term responders included patients who responded to gefitinib therapy for $>12$ months.

\section{Results}

EGFR mutations. The 44 NSCLC specimens included 43 adenocarcinomas and one large-cell neuroendocrine carcinoma. There was 1 patient with an exon 18 point mutation, 23 with an exon 19 deletion, 2 with an exon 20 point mutation, 16 with an exon 21 point mutation and 2 with both exon 20 and 21 point mutations (Table I).

Association of EGFR mutations with response to gefitinib. There were 4 non-responders, including the 2 patients with exon 20 mutation, 25 intermediate responders (including 10 patients under ongoing treatment) and 15 long-term responders ( 2 of whom are under ongoing treatment), including the 2 patients with both exon 20 and 21 mutations (Table II).

Immunostaining results. We investigated MET and HGF immunostaining in 4 non-responders, 3 of whom were MET-positive and HGF-negative, whereas 1 patient was MET-negative and HGF-positive (Fig. 1).

\section{Discussion}

Previous studies reported that the causes of acquired resistance to EGFR-TKIs in patients with EGFR mutations are a second mutation (T790M), MET amplification, or HGF-mediated MET activation. In those studies, $~ 50 \%$ of the cases with resistance to EGFR-TKIs exhibited a second mutation and $20 \%$ were due to MET amplification (8). Our results were similar to those of previous studies, where EGFR-TKI therapy was the initial treatment. However, in our study, patients with NSCLC and exon 20 mutation responded to gefitinib in the presence of an additional EGFR mutation. In particular, 2 cases (29 and 30) in this study were treated with gefitinib for 14 and 21 months, respectively. Our results were better in terms of PFS compared to those previously reported (2-13 months) (9-12).

Inukai et al reported that a small fraction of T790M-positive tumor cells at the beginning of treatment may lead to clinical gefitinib resistance as a result of the selective proliferation of T790M mutant cells (9). We therefore considered that the growth speed of T790M-positive cells and the number of T790M cells prior to EGFR-TKI treatment regulation were important for predicting PFS in patients with NSCLC and EGFR mutations. We considered that the T790M cell number was more important, rather than the T790M cell growth speed, as the latter is low (13). However, there is no established clinical method to quantitatively measure the number of T790M cells. Therefore, we must make a prediction based on the sensitivity of EGFR mutation testing in patients with NSCLC. The sensitivity of direct sequencing was previously found to be $\sim 25 \%$, that of cycleave PCR was $\sim 5 \%$ and that of Scorpion ARMS was $1 \%$ (14).

The PFS of NSCLC patients, as assessed by direct sequencing in a study by Wu et al was 2 months (11). However, the PFS of NSCLC patients assessed using cycleave PCR in our study was 17.5 months. We attributed the longer PFS in our study to the detection of fewer T790M cells in our patients using more sensitive cycleave PCR prior to EGFR-TKI treatment.

Consequently, our findings suggest that NSCLC patients may be long-term responders if a double mutation is identified using a highly sensitive method, such as cycleave PCR or Scorpion ARMS.

Our data and previous reports taken together, indicate that NSCLC with exon 20 mutation will respond to gefitinib treatment in the presence of an additional EGFR mutation. However, further investigations are required to determine the mechanism underlying our findings.

\section{References}

1. Lynch TJ, Bell DW, Sordella R, et al: Activating mutations in the epidermal growth factor receptor underlying responsiveness of non-small-cell lung cancer to gefitinib. N Engl J Med 350: 2129-2139, 2004

2. Paez JG, Janne PA, Lee JC, et al: EGFR mutations in lung cancer: correlation with clinical response to gefitinib therapy. Science 304: 1497-1500, 2004.

3. Inoue A, Suzuki T, Fukuhara T, et al: Prospective phase II study of gefitinib for chemotherapy-naive patients with advanced non-small-cell lung cancer with epidermal growth factor receptor gene mutations. J Clin Oncol 24: 3340-3346, 2006. 
4. Pao W, Miller VA, Politi KA, et al: Acquired resistance of lung adenocarcinomas to gefitinib or erlotinib is associated with a second mutation in the EGFR kinase domain. PLoS Med 2: e73, 2005.

5. Kobayashi S, Boggon TJ, Dayaram T, et al: EGFR mutation and resistance of non-small-cell lung cancer to gefitinib. N Engl J Med 352: 786-792, 2005.

6. Engelman JA, Zejnullahu K, Mitsudomi T, et al: MET amplification leads to gefitinib resistance in lung cancer by activating ERBB3 signaling. Science 316: 1039-1043, 2007.

7. Yano S, Wang W, Li Q, et al: Hepatocyte growth factor induces gefitinib resistance of lung adenocarcinoma with epiderma growth factor receptor-activating mutations. Cancer Res 68 : 9479-9487, 2008.

8. Nguyen KS, Kobayashi S and Costa DB: Acquired resistance to epidermal growth factor receptor tyrosine kinase inhibitors in non-small-cell lung cancers dependent on the epidermal growth factor receptor pathway. Clin Lung Cancer 10: 281-289, 2009.

9. Inukai M, Toyooka S, Ito S, et al: Presence of epidermal growth factor receptor gene $\mathrm{T} 790 \mathrm{M}$ mutation as a minor clone in non-small cell lung cancer. Cancer Res 66: 7854-7858, 2006.
10. Tokumo M, Toyooka S, Ichihara S, et al: Double mutation and gene copy number of EGFR in gefitinib refractory non-small-cell lung cancer. Lung cancer 53: 117-121, 2006.

11. Wu JY, Wu SG, Yang CH, et al: Lung cancer with epidermal growth factor receptor exon 20 mutations is associated with poor gefitinib treatment response. Clin Cancer Res 14: 4877-4882, 2008.

12. Su KY, Chen HY, Li KC, et al: Pretreatment epidermal growth factor receptor (EGFR) T790M mutation predicts shorter EGFR tyrosine kinase inhibitor response duration in patients with non-small-cell lung cancer. J Clin Oncol 30: 433-440, 2012.

13. Oxnard GR, Arcila ME, Chmielecki J, Ladanyi M, Miller VA and Pao W: New strategies in overcoming acquired resistance to epidermal growth factor receptor tyrosine kinase inhibitors in lung cancer. Clin Cancer Res 17: 5530-5537, 2011.

14. Pao W and Ladanyi M: Epidermal growth factor receptor mutation testing in lung cancer: searching for the ideal method. Clin Cancer Res 13: 4954-4955, 2007. 